\title{
Hardy-Sobolev type inequalities for generalized Baouendi-Grushin operators
}

\author{
Pengcheng Niu and Jingbo Dou
}




\title{
HARDY-SOBOLEV TYPE INEQUALITIES FOR GENERALIZED BAOUENDI-GRUSHIN OPERATORS
}

\author{
PENGCHENG NIU AND JINGBO DOU
}

Received 16 November, 2005

\begin{abstract}
In this paper we establish a class of Hardy-Sobolev type inequalities related to generalized Baouendi-Grushin operators. Our results contain the well-known Hardy type inequality and Sobolev type inequality for the class of operators. Furthermore, some new inequalities are obtained.
\end{abstract}

1991 Mathematics Subject Classification: 35H10, 35R45, 35J60

Keywords: Hardy-Sobolev type inequality, generalized Baouendi-Grushin operator

\section{INTRODUCTION}

In [6], a Hardy type inequality for the generalized Baouendi-Grushin vector fields

$$
Z_{i}=\frac{\partial}{\partial x_{i}}, \quad Z_{n+j}=|x|^{\alpha} \frac{\partial}{\partial y_{j}}, \quad(1 \leq i \leq n, 1 \leq j \leq m)
$$

where $x=\left(x_{1}, x_{2}, \ldots, x_{n}\right) \in \mathbb{R}^{n}, y=\left(y_{1}, y_{2} \ldots, y_{m}\right) \in \mathbb{R}^{m}, \alpha>0$, is given by

$$
\int_{\mathbb{R}^{n+m}} \frac{u^{2}}{d^{2}} \psi_{2 \alpha} d x d y \leq\left(\frac{2}{Q-2}\right)^{2} \int_{\mathbb{R}^{n+m}}\left|\nabla_{L} u\right|^{2} d x d y,
$$

for $u \in L^{2}\left(\mathbb{R}^{n+m}, \psi_{2 \alpha} d x d y\right)$ and $\left|\nabla_{L} u\right| \in L^{2}\left(\mathbb{R}^{n+m}\right)$, with $\nabla_{L}=\left(Z_{1}, \ldots, Z_{n}\right.$, $\left.Z_{n+1} \ldots, Z_{n+m}\right)$. Using (1.1), a unique continuation for the generalized BaouendiGrushin operator

$$
\mathcal{L}_{\alpha}=\Delta_{x}+|x|^{2 \alpha} \Delta_{y}=\sum_{i=1}^{n+m} Z_{i}=\nabla_{L} \cdot \nabla_{L}
$$

was proved. The proof of (1.1) used representation formulae of functions by the fundamental solution of $\mathcal{L}_{\alpha}$ at the origin.

The project was supported by National Science Basic Research Plan in Shaanxi Province of China, Grant No. 2006A09. 
The general version of (1.1) was established with a different approach in [10]. For any open subset $\Omega \subset \mathbb{R}^{n+m}$, D'Ambrosio obtained also the following Hardy type inequalities in [3]:

Let $p>1, n, m \geq 1$, and $\beta, \gamma \in \mathbb{R}$ such that $n+(1+\alpha) m>\gamma-\beta-p$ and $n>$ $\alpha p-\beta$. Then, for every $u \in D^{1, p}\left(\Omega,|x|^{\beta-\alpha p} d^{(1+\alpha) p-\gamma}\right)$ it follows

$$
c_{Q, p, \beta, \gamma}^{p} \int_{\Omega}|u|^{p} \frac{|x|^{\beta}}{d^{\gamma}} d x d y \leq \int_{\Omega}\left|\nabla_{L} u\right|^{p}|x|^{\beta-\alpha p} d^{(1+\alpha) p-\gamma} d x d y,
$$

where $c_{Q, p, \beta, \gamma}=\frac{Q+\beta-\gamma}{p}$. If $(0,0) \in \Omega$, then the constant (1.2) $c_{Q, p, \beta, \gamma}^{p}$ is sharp.

Recently, a Sobolev type inequality for the vector fields $Z_{1}, \ldots, Z_{n+m}$ (see [7]) states

$$
\left(\int_{\mathbb{R}^{n+m}}|u|^{2^{*}} d x d y\right)^{\frac{1}{2^{*}}} \leq S\left(\int_{\mathbb{R}^{n+m}}\left|\nabla_{L} u\right|^{2} d x d y\right)^{\frac{1}{2}},
$$

where $S$ is a positive constant, $2^{*}=\frac{2 Q}{Q-2}, Q=n+(\alpha+1) m$ is the homogeneous dimension with respect to the dilations

$$
\delta_{\lambda}(x, y)=\left(\lambda x, \lambda^{\alpha+1} y\right), \quad \lambda>0,(x, y) \in \mathbb{R}^{n+m},
$$

which is induced by $\mathcal{L}_{\alpha}$. Inequality (1.3) contains the result for $\alpha=1$ in [2].

We define the following distance from the origin on $\mathbb{R}^{n+m}$

$$
d(x, y)=\left(|x|^{2(\alpha+1)}+(\alpha+1)^{2}|y|^{2}\right)^{\frac{1}{2(\alpha+1)}} .
$$

It is easy to check that

$$
\begin{aligned}
\nabla_{L} d= & \frac{|x|^{\alpha}}{d^{2 \alpha+1}}\left(|x|^{\alpha} x_{1},|x|^{\alpha} x_{2}, \ldots,|x|^{\alpha} x_{n},\right. \\
& \left.(\alpha+1) y_{1},(\alpha+1) y_{2}, \ldots,(\alpha+1) y_{m}\right), \\
\left|\nabla_{L} d\right|^{2}= & \frac{|x|^{2 \alpha}}{d^{2 \alpha}}=\psi_{2 \alpha} .
\end{aligned}
$$

Let $C_{0}^{k}(\Omega)$ be the set of functions with compact in $C^{k}(\Omega)$ and $1<p<\infty$. We denote by $D^{1, p}(\Omega)$ the closure of $C_{0}^{\infty}(\Omega)$ under the norm $\left(\int_{\Omega}\left|\nabla_{L} u\right|^{p} d \xi\right)^{1 / p}$.

In this paper we will establish a class of Hardy-Sobolev type inequalities related to generalized Baouendi-Grushin operators. Our results contain the well-known Hardy type inequality and Sobolev type inequality for the class of operators.

This paper is organized as follows. In the next section, we prove a Hardy-Sobolev type inequality for $\mathcal{L}_{\alpha}$. This generalizes the inequalities in the Euclidean space in [1]. In Section 3, we give some new Hardy type inequalities on bounded domains. Our results include those of [3]. 


\section{HARDY-SOBOLEV INEQUALITIES}

In this section, set $(x, y)=\left(x^{\prime}, y^{\prime}, x^{\prime \prime}, y^{\prime \prime}\right)=\left(z_{1}, z_{2}\right) \in \mathbb{R}^{n+m}$ with $z_{1}=\left(x^{\prime}, y^{\prime}\right) \in$ $\mathbb{R}^{k+l} z_{2}=\left(x^{\prime \prime}, y^{\prime \prime}\right) \in \mathbb{R}^{n-k+(m-l)}, 1 \leq k \leq n, 1 \leq l \leq m$, and

$$
d_{1}=d\left(x^{\prime}, y^{\prime}\right)=\left(\left|x^{\prime}\right|^{2(\alpha+1)}+(\alpha+1)^{2}\left|y^{\prime}\right|^{2}\right)^{\frac{1}{2(\alpha+1)}} \text {. }
$$

We denote by $B_{R}^{\prime}(R)=\left\{\left(x^{\prime}, y^{\prime}\right) \in \mathbb{R}^{k+l} \mid d_{1}<R\right\}$ the open ball centered at $(0,0)$ with radius $R>0$, and put $\nabla_{L^{\prime}}=\left(Z_{1}, \ldots, Z_{k}, Z_{n+1}, \ldots, Z_{n+l}\right)$.

We note that the polar coordinate transformation defined in $[5,8]$ implies

$$
d z_{1}=d x^{\prime} d y^{\prime}=\rho^{k+(\alpha+1) l-1} d \rho d \sigma_{1},
$$

where $d \sigma_{1}=\left(\frac{1}{\alpha+1}\right)^{l}|\sin \theta|^{\frac{k}{\alpha+1}-1}|\cos \theta|^{l-1} d \theta d \omega_{k} d \omega_{l}$, and $\omega_{k}$ and $\omega_{l}$ are the Lebesgue measures of the unitary Euclidean spheres in $\mathbb{R}^{k}$ and $\mathbb{R}^{l}$, respectively.

The main inequalities in this section are the following.

Theorem 1 (Hardy-Sobolev type inequalities). Let us assume that satisfies the relations $0 \leq s \leq 2<k+(\alpha+1) l \leq Q$, and put

$$
2 *(s)=\frac{2(Q-s)}{Q-2} .
$$

Then there exists a positive constant $C(s, \alpha, k, l)$ such that for every $u \in D^{1,2}\left(\mathbb{R}^{n+m}\right)$

$$
\int_{\mathbb{R}^{n+m}} \frac{\left|x^{\prime}\right|^{s \alpha}}{d_{1}^{s \alpha}} \frac{|u|^{2 *(s)}}{d_{1}^{s}} d x d y \leq C\left(\int_{\mathbb{R}^{n+m}}\left|\nabla_{L} u\right|^{2} d x d y\right)^{\frac{Q-s}{\mathcal{Q}-2}},
$$

where $D^{1,2}\left(\mathbb{R}^{n+m}\right)$ is the completion of $C_{0}^{\infty}\left(\mathbb{R}^{n+m}\right)$ under the norm

$$
\|u\|=\left(\int_{\mathbb{R}^{n+m}}\left|\nabla_{L} u\right|^{2} d x d y\right)^{\frac{1}{2}} .
$$

Remark 1. If $s=0, k=n, l=m$, then (2.2) is (1.3); if $s=2, k=n, l=m$, then (2.2) is (1.1).

We prove first a lemma, which gives a representation formula of functions only depending on vector fields $\left(Z_{1}, \ldots, Z_{n+m}\right)$ and dilation of $\mathcal{L}_{\alpha}$. It establishes the connection between the function $u$ and its generalized gradient $\nabla_{L} u$.

Lemma 1. For any $u(x, y) \in C_{0}^{\infty}\left(\mathbb{R}^{n+m}\right)$, we have

$$
u(x, y)=-\int_{1}^{\infty}\left[\frac{1}{\lambda|x|^{2 \alpha}}\left\langle\nabla_{L} u, \nabla_{L}\left(\frac{d^{2(\alpha+1)}}{2(\alpha+1)}\right)\right\rangle\right] \circ \delta_{\lambda} d \lambda .
$$

Proof. Clearly,

$$
\begin{aligned}
& \nabla_{L}\left(d^{2(\alpha+1)}\right)=2(\alpha+1) d^{2 \alpha+1} \nabla_{L} d=2(\alpha+1)|x|^{\alpha} \\
& \quad\left(|x|^{\alpha} x_{1},|x|^{\alpha} x_{2}, \ldots,|x|^{\alpha} x_{n},(\alpha+1) y_{1},(\alpha+1) y_{2}, \ldots,(\alpha+1) y_{m}\right) .
\end{aligned}
$$


By (1.4) and (2.4), one has

$$
\begin{aligned}
\frac{d}{d \lambda} u \circ \delta_{\lambda} & =\frac{d u\left(\delta_{\lambda}(x, y)\right)}{d \lambda} \\
& =\left[\sum_{i=1}^{n} x_{i} \frac{\partial u}{\partial x_{i}} \circ \delta_{\lambda}(x, y)+(\alpha+1) \sum_{j=1}^{m} \lambda^{\alpha} y_{j} \frac{\partial u}{\partial y_{j}} \circ \delta_{\lambda}(x, y)\right] \\
& =\left[\sum_{i=1}^{n} \frac{x_{i}}{\lambda} \frac{\partial u}{\partial x_{i}}+(\alpha+1) \sum_{j=1}^{m} \frac{y_{j}}{\lambda} \frac{\partial u}{\partial y_{j}}\right] \circ \delta_{\lambda}(x, y) \\
& =\left[\frac{1}{\lambda|x|^{2 \alpha}}\left(\sum_{i=1}^{n}|x|^{2 \alpha} x_{i} \frac{\partial u}{\partial x_{i}}+(\alpha+1) \sum_{j=1}^{m}|x|^{2 \alpha} y_{j} \frac{\partial u}{\partial y_{j}}\right)\right] \circ \delta_{\lambda}(x, y) \\
& =\left[\frac{1}{\lambda|x|^{2 \alpha}}\left\langle\nabla_{L} u, \nabla_{L}\left(\frac{d^{2(\alpha+1)}}{2(\alpha+1)}\right)\right\rangle \circ \delta_{\lambda}(x, y)\right.
\end{aligned}
$$

Therefore, we obtain

$$
\begin{aligned}
u(x, y) & =-\int_{1}^{\infty} \frac{d}{d \lambda}\left(u\left(\delta_{\lambda}(x, y)\right)\right) d \lambda \\
& =-\int_{1}^{\infty}\left[\frac{1}{\lambda|x|^{2 \alpha}}\left\langle\nabla_{L} u, \nabla_{L}\left(\frac{d^{2(\alpha+1)}}{2(\alpha+1)}\right)\right\rangle\right] \delta_{\lambda} d \lambda,
\end{aligned}
$$

as required.

Remark 2. We note that $\nabla_{L}\left(d^{2(\alpha+1)}\right)=2(\alpha+1) d^{2 \alpha+1} \nabla_{L} d$ in the proof above, and so

$$
\begin{aligned}
\left|\nabla_{L}\left(d^{2(\alpha+1)}\right)\right|^{2} & =2\left[(\alpha+1) d^{2 \alpha+1}\right]^{2}\left|\nabla_{L} d\right|^{2} \\
& =[2(\alpha+1)]^{2}|x|^{2 \alpha} d^{2(\alpha+1)} .
\end{aligned}
$$

Proof of Theorem 1. We need only to consider the cases where $u \geq 0$ and $u \in$ $C_{0}^{\infty}\left(\mathbb{R}^{n+m}\right)$. Introduce the notation $S_{1}=\partial B_{1}^{\prime}=\left\{\left(x^{\prime}, y^{\prime}\right) \in \mathbb{R}^{k+l}\left(x^{\prime}, y^{\prime}\right)=1\right\}$ and $\vartheta=\left(\tau_{1}, \tau_{2}\right)=\left(\tau_{11}, \ldots, \tau_{1 k}, \tau_{21}, \ldots, \tau_{2 k}\right) \in S_{1}$. We introduce the transformation

$$
z_{1}=\left(x^{\prime}, y^{\prime}\right)=(\rho, \vartheta),
$$


where $\rho=d_{1}, \vartheta=\left(\tau_{1}, \tau_{2}\right)=\delta_{\frac{1}{\rho}}\left(x^{\prime}, y^{\prime}\right)$. By Lemma 1 , we get

$$
\begin{aligned}
& \int_{\mathbb{R}^{n+m}} \frac{\left|x^{\prime}\right|^{s \alpha}}{\rho^{s \alpha}} \frac{|u|^{2 *}}{\rho^{s}} d x d y=\int_{\mathbb{R}^{n-k+(m-l)}} d z_{2} \int_{\mathbb{R}^{k+l}} \frac{\left|x^{\prime}\right|^{s \alpha}}{\rho^{s \alpha}} \frac{|u|^{2 *}}{\rho^{s}} d z_{1} \\
& =-\int_{1}^{\infty} d \lambda \int_{\mathbb{R}^{n-k+(m-l)}} d z_{2} \\
& \times \int_{\mathbb{R}^{k+l}}\left[\frac{\left|x^{\prime}\right|^{s \alpha}}{\rho^{s \alpha+s}} \frac{1}{\lambda\left|x^{\prime}\right|^{2 \alpha}}\left\langle\nabla_{L^{\prime}} u^{2 *}, \nabla_{L^{\prime}}\left(\frac{\rho^{2(\alpha+1)}}{2(\alpha+1)}\right)\right\rangle\right] \circ \delta_{\lambda}\left(x^{\prime}, y^{\prime}\right) d z_{1} \text {. }
\end{aligned}
$$

Putting

$$
F=\frac{1}{\left|x^{\prime}\right|^{2 \alpha}}\left\langle\nabla_{L^{\prime}} u^{2 *}, \nabla_{L^{\prime}}\left(\frac{\rho^{2(\alpha+1)}}{2(\alpha+1)}\right)\right\rangle,
$$

we obtain from (2.1) that

$$
\begin{aligned}
\int_{\mathbb{R}^{n+m}} & \frac{\left|x^{\prime}\right|^{s \alpha}}{\rho^{s \alpha}} \frac{|u|^{2 *}}{\rho^{s}} d x d y \\
= & -\int_{1}^{\infty} d \lambda \int_{\mathbb{R}^{n-k+(m-l)}} d z_{2} \int_{\mathbb{R}^{k+l}} \frac{\left|x^{\prime}\right|^{s \alpha}}{\rho^{s \alpha+s}} \frac{F}{\lambda} \circ \delta_{\lambda}\left(x^{\prime}, y^{\prime}\right) d z_{1} \\
= & -\int_{1}^{\infty} d \lambda \int_{\mathbb{R}^{n-k+(m-l)}} d z_{2} \int_{S_{1}} d \sigma_{1} \int_{0}^{\infty} \frac{\rho^{s \alpha}\left|\tau_{1}\right|^{s \alpha}}{\rho^{s \alpha+s}} \frac{F}{\lambda} \circ \delta_{\lambda \rho}(\vartheta) \rho^{k+(\alpha+1) l-1} d \rho \\
= & -\int_{1}^{\infty} \lambda^{-(k+(\alpha+1) l-1-s)-2} d \lambda \int_{\mathbb{R}^{n-k+(m-l)}} d z_{2} \\
& \times \int_{S_{1}} d \sigma_{1} \int_{0}^{\infty}\left|\tau_{1}\right|^{s \alpha} F \circ \delta_{r}(\vartheta) r^{k+(\alpha+1) l-1-s} d r \quad(\lambda \rho=r) \\
= & -\frac{1}{k+(\alpha+1) l-s} \int_{\mathbb{R}^{n-k+(m-l)}} d z_{2} \int_{\mathbb{R}^{k+l}} \frac{\left|x^{\prime}\right|^{s \alpha}}{\rho^{s \alpha+s}} F d z_{1} \\
= & -\frac{1}{k+(\alpha+1) l-s} \int_{\mathbb{R}^{n-k+(m-l)}} d z_{2} \\
& \times \int_{\mathbb{R}^{k+l}} \frac{\left|x^{\prime}\right|^{s \alpha}}{\rho^{s \alpha+s}}\left[\frac{1}{\left|x^{\prime}\right|^{2 \alpha}}\left\langle\nabla_{L^{\prime} u^{2 *}}, \nabla_{L^{\prime}}\left(\frac{\rho^{2(\alpha+1)}}{2(\alpha+1)}\right)\right\rangle\right] d z_{1}
\end{aligned}
$$

Now we consider the cases $1 \leq s \leq 2$ and $0<s<1$, respectively. 
Case 1: $1 \leq s \leq 2$. If $1<s<2$, then (2.6), (2.9) and Hölder's inequality yield

$$
\begin{aligned}
& \int_{\mathbb{R}^{n+m}} \frac{\left|x^{\prime}\right|^{s \alpha}}{\rho^{s \alpha}} \frac{|u|^{2 *}}{\rho^{s}} d x d y \\
& \leq \frac{1}{k+(\alpha+1) l-s} \int_{\mathbb{R}^{n-k+(m-l)}} d z_{2} \int_{\mathbb{R}^{k+l}} \frac{\left|x^{\prime}\right|^{s \alpha-2 \alpha}}{\rho^{s \alpha+s}}\left|\nabla_{L^{\prime}} u^{2 *}\right| \frac{\left|\nabla_{L^{\prime}}\left(\rho^{2(\alpha+1)}\right)\right|}{2(\alpha+1)} d z_{1} \\
& =\frac{2 *}{k+(\alpha+1) l-s} \int_{\mathbb{R}^{n+m}} \frac{\left|x^{\prime}\right|^{s \alpha-2 \alpha}}{\rho^{s \alpha+s}} u^{2_{*}-1}\left|\nabla_{L^{\prime}} u\right|\left|x^{\prime}\right|^{\alpha} \rho^{\alpha+1} d x d y \\
& =\frac{2 *}{k+(\alpha+1) l-s} \int_{\mathbb{R}^{n+m}} \frac{\left|x^{\prime}\right|^{\alpha(s-1)}}{\rho^{\alpha(s-1)+s-1}} u^{2_{*}-1}\left|\nabla_{L^{\prime}} u\right| d x d y \\
& \leq \frac{2 *}{k+(\alpha+1) l-s} \int_{\mathbb{R}^{n+m}} \frac{\left|x^{\prime}\right|^{\alpha(s-1)}}{\rho^{\alpha(s-1)}} \frac{u^{2 *} \frac{s-1}{\rho^{s-1}}}{\rho^{s-1}} u^{2 * \frac{1}{s}-1}\left|\nabla_{L} u\right| d x d y \\
& \left.\quad \times\left(\int_{\mathbb{R}^{n+m}}\left|\nabla_{L} u\right|^{2} d x d y\right)^{\frac{s-1}{2}} \cdot \frac{\left|x^{\prime}\right|^{s \alpha}}{\rho^{s \alpha}} \frac{u^{2 *}}{\rho^{s}} d x d y\right)^{\frac{s-1}{s}}\left(\int_{\mathbb{R}^{n+m}} u^{2^{*}} d x d y\right)^{\frac{2-s}{2 s}}
\end{aligned}
$$

By the Sobolev type inequality (1.3) we have

$$
\begin{aligned}
\int_{\mathbb{R}^{n+m}} & \frac{\left|x^{\prime}\right|^{s \alpha}}{\rho^{s \alpha}} \frac{|u|^{2 *}}{\rho^{s}} d x d y \\
& \leq\left(\frac{2 *}{k+(\alpha+1) l-s}\right)^{s}\left(\int_{\mathbb{R}^{n+m}} u^{2^{*}} d x d y\right)^{\frac{2-s}{2}}\left(\int_{R^{n+m}}\left|\nabla_{L} u\right|^{2} d x d y\right)^{\frac{s}{2}} \\
& \leq\left(\frac{2 *}{k+(\alpha+1) l-s}\right)^{s} S^{\frac{2^{*}(2-s)}{2}}\left(\int_{\mathbb{R}^{n+m}}\left|\nabla_{L} u\right|^{2} d x d y\right)^{\frac{s}{2}+\frac{2^{*}}{2} \cdot \frac{2-s}{2}} \\
& =\left(\frac{2 *}{k+(\alpha+1) l-s}\right)^{s} S^{\frac{2^{*}(2-s)}{2}}\left(\int_{\mathbb{R}^{n+m}}\left|\nabla_{L} u\right|^{2} d x d y\right)^{\frac{Q-s}{Q-2}}
\end{aligned}
$$

For $s=1$, relation (2.10) leads one to

$$
\int_{\mathbb{R}^{n+m}} \frac{\left|x^{\prime}\right|^{\alpha}}{\rho^{\alpha}} \frac{|u|^{2 *(1)}}{\rho} d x d y \leq \frac{2 *(1)}{k+(\alpha+1) l-1} S^{\frac{2^{*}}{2}}\left(\int_{\mathbb{R}^{n+m}}\left|\nabla_{L} u\right|^{2} d x d y\right)^{\frac{Q-1}{Q-2}}
$$

where $2_{*}(1)=\frac{2(Q-1)}{Q-2}$. 
If $s=2$, then $2_{*}=2$ and by (2.10), it follows

$$
\begin{aligned}
& \int_{\mathbb{R}^{n+m}} \frac{\left|x^{\prime}\right|^{2 \alpha}}{\rho^{2 \alpha}} \frac{|u|^{2}}{\rho^{2}} d x d y \leq \frac{2}{k+(\alpha+1) l-2} \int_{\mathbb{R}^{n+m}} \frac{\left|x^{\prime}\right|^{\alpha}}{\rho^{\alpha+1}} u\left|\nabla_{L} u\right| d x d y \\
\leq & \frac{2}{k+(\alpha+1) l-2}\left(\int_{\mathbb{R}^{n+m}}\left(\frac{\left|x^{\prime}\right|^{\alpha} u}{\rho^{\alpha+1}}\right)^{2} d x d y\right)^{\frac{1}{2}}\left(\int_{\mathbb{R}^{n+m}}\left|\nabla_{L} u\right|^{2} d x d y\right)^{\frac{1}{2}},
\end{aligned}
$$

namely,

$$
\int_{\mathbb{R}^{n+m}} \frac{\left|x^{\prime}\right|^{2 \alpha}}{\rho^{2 \alpha}} \frac{|u|^{2}}{\rho^{2}} d x d y \leq\left(\frac{2}{k+(\alpha+1) l-2}\right)^{2} \int_{\mathbb{R}^{n+m}}\left|\nabla_{L} u\right|^{2} d x d y .
$$

Case 2: $0<s<1$. Using

$$
2 *(s)=\frac{2(Q-s)}{Q-2}=(1-s) 2^{*}+s 2_{*}(1)
$$

and (2.12), we have

$$
\begin{aligned}
\int_{\mathbb{R}^{n+m}} \frac{\left|x^{\prime}\right|^{s \alpha}}{\rho^{s \alpha}} \frac{|u|^{2 *}}{\rho^{s}} d x d y=\int_{\mathbb{R}^{n+m}}|u|^{(1-s) 2^{*}}\left(\frac{\left|x^{\prime}\right|^{\alpha} u^{2 *(1)}}{\rho^{\alpha+1}}\right)^{s} d x d y \\
\leq\left(\int_{\mathbb{R}^{n+m}}|u|^{2^{*}} d x d y\right)^{1-s}\left(\int_{\mathbb{R}^{n+m}} \frac{\left|x^{\prime}\right|^{\alpha}}{\rho^{\alpha}} \frac{|u|^{2 *(1)}}{\rho} d x d y\right)^{s} \\
\leq S^{2^{*}(1-s)}\left(\int_{\mathbb{R}^{n+m}}\left|\nabla_{L} u\right|^{2} d x d y\right)^{\frac{2^{*}(1-s)}{2}}\left(\frac{2 *(1)}{k+(\alpha+1) l-1} S^{\frac{2^{*}}{2}}\right)^{s} \\
\left.\quad \times\left(\int_{\mathbb{R}^{n+m}}\left|\nabla_{L} u\right|^{2} d x d y\right)^{\frac{(Q-1) s}{Q-2}}\right)^{s}\left(\int_{\mathbb{R}^{n+m}}\left|\nabla_{L} u\right|^{2} d x d y\right)^{\frac{Q-s}{Q-2}},
\end{aligned}
$$

and the proof is complete.

\section{HARDY TYPE INEQUALITIES ON BOUNDED DOMAINS}

In this section, let $\Omega \subset \mathbb{R}^{n+m}$ denote any bounded domain, $(x, y)=\left(z_{1}, z_{2}\right) \in \Omega$, $d_{1}=d\left(x^{\prime}, y^{\prime}\right), B_{R}^{\prime}(R), \nabla_{L^{\prime}}$ as before. Define

$$
\begin{aligned}
r_{\epsilon} & :=\left(\epsilon^{2}+\left|x^{\prime}\right|^{2}\right)^{\frac{1}{2}}, \\
\sigma_{\epsilon} & :=\left(\begin{array}{cc}
I_{k} & 0 \\
0 & r_{\epsilon}^{\alpha} I_{l}
\end{array}\right),
\end{aligned}
$$


and set $\sigma_{\epsilon} \nabla=\nabla_{L^{\prime}}^{\epsilon}$. For any vector field $h \in C^{1}\left(\Omega_{1}, \mathbb{R}^{k+l}\right)$, we shall write $\operatorname{div}_{L^{\prime}}^{\epsilon}=$ $\operatorname{div}\left(\sigma_{\epsilon} h\right)$.

The following is the main result of this section.

Theorem 2. Let $p>1, k, l \geq 1$, and $\beta, \gamma \in \mathbb{R}$, be such that $k+(\alpha+1) l>\gamma-$ $\beta-p$ and $k>\alpha p-\beta$. Then, for any $u \in D^{1, p}\left(\Omega,\left|x^{\prime}\right|^{\beta-\alpha p} d_{1}^{(\alpha+1) p-\gamma}\right)$, we get

$$
c_{k, l, p, \beta, \gamma}^{p} \int_{\Omega}|u|^{p} \frac{\left|x^{\prime}\right|^{\beta}}{d_{1}^{\gamma}} d x d y \leq \int_{\Omega}\left|\nabla_{L} u\right|^{p}\left|x^{\prime}\right|^{\beta-\alpha p} d_{1}^{(\alpha+1) p-\gamma} d x d y,
$$

where $c_{k, l, p, \beta, \gamma}=\frac{k+(\alpha+1) l+\beta-\gamma}{p}$.

If $(0,0) \in \Omega$, then the constant $c_{k, l, p, \beta, \gamma}^{p}$ in (3.1) is sharp.

Corollary 1. If $1<p<k+(\alpha+1) l$, then

$$
\begin{aligned}
& c_{k, l, p}^{p} \int_{\Omega} \frac{\left|x^{\prime}\right|^{\alpha p}}{d_{1}^{\alpha p}} \frac{|u|^{p}}{d_{1}^{p}} d x d y \leq \int_{\Omega}\left|\nabla_{L} u\right|^{p} d x d y \\
& \qquad \text { for } u \in D^{1, p}(\Omega) ; \\
& c_{k, l, p}^{p} \int_{\Omega} \frac{|u|^{p}}{d_{1}^{p}} d x d y \leq \int_{\Omega}\left|\nabla_{L} u\right|^{p} \frac{\left|x^{\prime}\right|^{\alpha p}}{d_{1}^{\alpha p}} d x d y, \\
& \qquad \text { for } k>\alpha p, u \in D^{1, p}\left(\Omega, \frac{\left|x^{\prime}\right|^{\alpha p}}{d_{1}^{\alpha p}}\right) ;
\end{aligned}
$$

and

$$
\begin{aligned}
& c_{k, l, p}^{p} \int_{\Omega} \frac{|u|^{p}}{d_{1}^{p}} d x d y \leq \int_{\Omega}\left|\nabla_{L} u\right|^{p} \frac{\left|x^{\prime}\right|^{(\alpha+1) p}}{d_{1}^{(\alpha+1) p}} d x d y \\
& \qquad \text { for } k>(\alpha+1) p, u \in D^{1, p}\left(\Omega, \frac{\left|x^{\prime}\right|^{(\alpha+1) p}}{d_{1}^{(\alpha+1) p}}\right),
\end{aligned}
$$

where $c_{k, l, p}=\frac{k+(\alpha+1) l-p}{p}$.

In order to prove our results, we will use the following statement in [3].

Proposition 1. Let $\epsilon>0$, and $h \in C^{1}\left(\Omega_{1}, \mathbb{R}^{k+l}\right)$ such that $\operatorname{div}_{L^{\prime}}^{\epsilon} h>0$. Then for any $p>1$ and $u \in C_{0}^{1}\left(\Omega, \mathbb{R}^{n+m}\right)$, one has

$$
\int_{\Omega_{1}}|u|^{p} \operatorname{div}_{L^{\prime}}^{\epsilon} h d z_{1} \leq p^{p} \int_{\Omega_{1}}|h|^{p}\left|\operatorname{div}_{L^{\prime}}^{\epsilon} h\right|^{-(p-1)}\left|\nabla_{L^{\prime}}^{\epsilon} u\right| d z_{1} .
$$

The following proposition is also useful. For a similar description in the Euclidean space see [9]. 
Proposition 2. If $\omega \in C_{0}^{\infty}\left(\mathbb{R}^{n+m}\right)$, then

$$
\inf _{\omega \in C_{0}^{\infty}\left(\mathbb{R}^{n+m}\right), \omega \neq 0} \frac{\int_{\mathbb{R}^{n+m}}\left|\nabla_{L} \omega\right|^{p} d x d y}{\int_{\mathbb{R}^{n+m}}|\omega|^{p} d x d y}=0 .
$$

Proof. Set $\varphi \in C_{0}^{\infty}\left(\mathbb{R}^{n+m}\right)$ to be a nonnegative radial function and satisfy

$$
\varphi(d)= \begin{cases}1 & \text { if } d \leq \frac{1}{2} \\ 0 & \text { if } d \geq 1\end{cases}
$$

with $\int_{\mathbb{R}^{n+m}} \varphi^{p} d x d y=1$. Letting $\epsilon<1$ and putting $\varphi_{\epsilon}(d)=\epsilon^{-\frac{Q}{p}} \varphi\left(\frac{d}{\epsilon}\right)$, we get $\int_{\mathbb{R}^{n+m}} \varphi_{\epsilon}(d)^{p} d x d y=1$. Note that $\nabla_{L} \varphi_{\epsilon}(d)=\epsilon^{-\frac{Q}{p}-1} \varphi^{\prime}\left(\frac{d}{\epsilon}\right) \nabla_{L} d$. Setting $d=\epsilon \rho$ and using (2.1) in $\mathbb{R}^{n+m}$, we get

$$
\begin{aligned}
\int_{\mathbb{R}^{n+m}}\left|\nabla_{L} \varphi_{\epsilon}(d)\right|^{p} d x d y & =\epsilon^{-Q-p} \int_{\mathbb{R}^{n+m}} \psi_{p \alpha}\left|\varphi^{\prime}\left(\frac{d}{\epsilon}\right)\right|^{p} d x d y \\
& =\epsilon^{-p} s_{n, m} \int_{\{\rho<1\}} \rho^{Q-1}\left|\varphi^{\prime}(\rho)\right|^{p} d \rho \longrightarrow 0 \quad(\epsilon \rightarrow \infty)
\end{aligned}
$$

where

$$
s_{n, m}=\left(\frac{1}{\alpha+1}\right)^{m} \omega_{n} \omega_{m} \int_{a_{1}}^{a_{2}}|\sin \theta|^{\frac{n+p \alpha}{\alpha+1}-1}|\cos \theta|^{m-1} d \theta .
$$

This completes the proof.

Proof of Theorem 2. Without loss of generality, we shall consider a smooth function $u \in C_{0}^{\infty}(\Omega)$. The general case will follow by the density argument. For $\epsilon>0$, define

$$
d_{1, \epsilon}=\left(r_{\epsilon}^{2(\alpha+1)}+(\alpha+1)^{2}\left|y^{\prime}\right|^{2}\right)^{\frac{1}{2(\alpha+1)}}
$$

and

$$
h_{\epsilon}^{1}=\frac{1}{d_{1, \epsilon}^{\gamma}}\left(\begin{array}{c}
x^{\prime} r_{\epsilon}^{\beta} \\
(\alpha+1)\left|x^{\prime}\right|^{2} y^{\prime} r_{\epsilon}^{\beta-\alpha-2}
\end{array}\right) .
$$

A simple computation shows that

$$
\begin{aligned}
\operatorname{div}_{L^{\prime}}^{\epsilon} h_{\epsilon}^{1} & =\operatorname{div} \frac{1}{d_{1, \epsilon}^{\gamma}}\left(\begin{array}{c}
x^{\prime} r_{\epsilon}^{\beta} \\
(\alpha+1)\left|x^{\prime}\right|^{2} y^{\prime} r_{\epsilon}^{\beta-2}
\end{array}\right) \\
& =\frac{r_{\epsilon}^{\beta}}{d_{1, \epsilon}^{\gamma}}\left(k+((\alpha+1) l+\beta-\gamma) \frac{\left|x^{\prime}\right|^{2}}{r_{\epsilon}^{2}}\right)
\end{aligned}
$$


and

$$
\left|h_{\epsilon}^{1}\right|=\frac{r_{\epsilon}^{\beta-\alpha-2}\left|x^{\prime}\right|^{2}}{d_{1, \epsilon}^{\gamma}}\left(\frac{r_{\epsilon}^{2 \alpha+4}}{\left|x^{\prime}\right|^{2}}+(\alpha+1)^{2}\left|y^{\prime}\right|^{2}\right)^{\frac{1}{2}} .
$$

Putting $f_{\epsilon}(s)=k+((\alpha+1) l+\beta-\gamma) \frac{|s|^{2}}{\epsilon^{2}+s^{2}}, s \geq 0$, it is easy to see that

$$
f_{\epsilon}(s) \geq \begin{cases}k & \text { if }(\alpha+1) l+\beta-\gamma \geq 0, \\ k+(\alpha+1) l+\beta-\gamma & \text { if }(\alpha+1) l+\beta-\gamma<0,\end{cases}
$$

for every $\epsilon>0$ and $s \geq 0$. Since $r_{\epsilon} \geq \epsilon$, if $k+(\alpha+1) l>\gamma-\beta$, then $\operatorname{div}_{L^{\prime}}^{\epsilon} h_{\epsilon}^{1}>0$.

Now we choose $h=h_{\epsilon}^{1}$ in (3.5) and obtain

$$
\begin{aligned}
& \int_{\Omega}|u|^{p} \frac{r_{\epsilon}^{\beta}}{d_{1, \epsilon}^{\gamma}} f_{\epsilon}\left(\left|x^{\prime}\right|\right) d x d y=\int_{\Omega_{2}} d z_{2} \int_{\Omega_{1}}|u|^{p} \frac{r_{\epsilon}^{\beta}}{d_{1, \epsilon}^{\gamma}} f_{\epsilon}\left(\left|x^{\prime}\right|\right) d z_{1} \\
& \leq\left. p^{p} \int_{\Omega_{2}} d z_{2} \int_{\Omega_{1}}\left|h_{\epsilon}^{1}\right|^{p}\left|\frac{r_{\epsilon}^{\beta}}{d_{1, \epsilon}^{\gamma}} f_{\epsilon}\left(\left|x^{\prime}\right|\right)^{-(p-1)}\right| \nabla_{L^{\prime}}^{\epsilon} u\right|^{p} d z_{1} \\
& =p^{p} \int_{\Omega_{2}} d z_{2} \int_{\Omega_{1}}\left|\nabla_{L^{\prime}}^{\epsilon} u\right|^{p} \frac{r_{\epsilon}^{-\beta(p-1)}\left(r_{\epsilon}^{\beta-\alpha-2}\left|x^{\prime}\right|^{2}\right)^{p}\left(\frac{r_{\epsilon}^{2 \alpha+4}}{\left|x^{\prime}\right|^{2}}+(\alpha+1)^{2}\left|y^{\prime}\right|^{2}\right)^{\frac{p}{2}}}{d_{1, \epsilon}^{\gamma p} d_{1, \epsilon}^{-\gamma(p-1)} f_{\epsilon}\left(\left|x^{\prime}\right|\right)^{p-1}} d z_{1} \\
& =p^{p} \int_{\Omega_{2}} d z_{2} \int_{\Omega_{1}}\left|\nabla_{L^{\prime}}^{\epsilon} u\right|^{p} \frac{r_{\epsilon}^{\beta-(2+\alpha) p}\left|x^{\prime}\right|^{2 p}\left(\frac{r_{\epsilon}^{2 \alpha+4}}{\left|x^{\prime}\right|^{2}}+(\alpha+1)^{2}\left|y^{\prime}\right|^{2}\right)^{\frac{p}{2}}}{d_{1, \epsilon}^{\gamma} f_{\epsilon}\left(\left|x^{\prime}\right|\right)^{p-1}} d z_{1} .
\end{aligned}
$$

Let $m_{1}=\min \{k, k+(\alpha+1) l+\beta-\gamma\}>0$. By $r<r_{\epsilon}$ and (3.9) the right-hand side of (3.10) can be estimated as follows:

$$
\begin{aligned}
\left|\nabla_{L^{\prime}}^{\epsilon} u\right|^{p} \frac{r_{\epsilon}^{\beta-(2+\alpha) p}\left|x^{\prime}\right|^{2 p}\left(\frac{r_{\epsilon}^{2 \alpha+4}}{\left|x^{\prime}\right|^{2}}+(\alpha+1)^{2}\left|y^{\prime}\right|^{2}\right)^{\frac{p}{2}}}{d_{1, \epsilon}^{\gamma} f_{\epsilon}\left(\left|x^{\prime}\right|\right)^{p-1}} & \\
& \leq \frac{\left|\nabla_{L^{\prime}}^{\epsilon} u\right|^{p}}{m_{1}^{p-1}} r_{\epsilon}^{\beta-\alpha p} d_{1, \epsilon}^{(\alpha+1) p-\gamma} .
\end{aligned}
$$


Therefore, following the assumptions $k+(\alpha+1) l>\gamma-\beta-p$ and $k>\alpha p-\beta$, and applying the Lebesgue dominated convergence theorem to (3.10), we have

$$
\begin{aligned}
\int_{\Omega}|u|^{p} \frac{\left|x^{\prime}\right|^{\beta}}{d_{1}^{\gamma}} f\left(\left|x^{\prime}\right|\right) d x d y & =\lim _{\epsilon \rightarrow 0} \int_{\Omega}|u|^{p} \frac{r_{\epsilon}^{\beta}}{d_{1, \epsilon}^{\gamma}} f_{\epsilon}\left(\left|x^{\prime}\right|\right) d x d y \\
& \leq \lim _{\epsilon \rightarrow 0} p^{p} \int_{\Omega_{2}} d z_{2} \int_{\Omega_{1}} \frac{\left|\nabla_{L^{\prime}}^{\epsilon} u\right|^{p}}{m_{1}^{p-1}} r_{\epsilon}^{\beta-\alpha p} d_{1, \epsilon}^{(\alpha+1) p-\gamma} d z_{1} \\
& \leq p^{p} \int_{\Omega} \frac{\left|\nabla_{L} u\right|^{p}}{m_{1}^{p-1}}\left|x^{\prime}\right|^{\beta-\alpha p} d_{1}^{(\alpha+1) p-\gamma} d x d y
\end{aligned}
$$

This shows the claim of (3.1).

Choosing $(\beta, \gamma)=(\alpha p,(\alpha+1) p),(\beta, \gamma)=(0, p)$ and $(\beta, \gamma)=(-p, 0)$ in (3.1), implies the inequalities (3.2), (3.3) and (3.4), respectively.

Next, if $(0,0) \in \Omega$, we prove the constant $c_{k, l, p, \beta, \gamma}^{p}$ is sharp. The approach here comes from that in [9].

Let $C(\Omega)$ be the best constant in (3.1), that is

$$
C(\Omega)=\inf _{\phi \in C_{0}^{\infty}(\Omega), \phi \neq 0} \frac{\int_{\Omega}\left|\nabla_{L} \phi\right|^{p}\left|x^{\prime}\right|^{\beta-p \alpha} d_{1}^{(\alpha+1) p-\gamma} d x d y}{\int_{\Omega}|\phi|^{p} \frac{\left|x^{\prime}\right|^{\beta}}{d_{1}^{\gamma}} d x d y} .
$$

From (3.1), we easily get $C(\Omega) \geq c_{k, l, p, \beta, \gamma}^{p}$. We shall prove the equality sign holds.

Put $\phi \in C_{0}^{\infty}(\Omega)$ and $\phi=v\left(z_{1}\right) \omega\left(z_{2}\right)$, where $v\left(z_{1}\right) \in C_{0}^{\infty}\left(\mathbb{R}^{k+l} \backslash\{(0,0)\}\right)$, $\omega\left(z_{2}\right) \in C_{0}^{\infty}\left(\mathbb{R}^{n-k+(m-l)}\right)$. By the convexity of the function $\left(a^{2}+b^{2}\right)^{\frac{p}{2}}$ for $a, b \geq$ 0 , we have

$$
\left(a^{2}+b^{2}\right)^{\frac{p}{2}} \leq(1-\lambda)^{1-p} a^{p}+\lambda^{1-p} b^{p}
$$

where $p>1,0<\lambda<1$. Therefore, we find

$$
\begin{aligned}
\left|\nabla_{L} \phi\right|^{p} & =\left|\left(\nabla_{L}\left(v\left(z_{1}\right) \omega\left(z_{2}\right)\right)\right)^{2}\right|^{\frac{p}{2}} \\
& =\left(\left|\nabla_{L^{\prime}} v\right|^{2} \omega^{2}+v^{2}\left|\nabla_{L^{\prime \prime}} \omega\right|^{2}\right)^{\frac{p}{2}} \\
& \leq(1-\lambda)^{1-p}\left|\nabla_{L^{\prime}} v\right|^{p} \omega^{p}+\lambda^{1-p} v^{p}\left|\nabla_{L^{\prime \prime}} \omega\right|^{p},
\end{aligned}
$$


where $\nabla_{L^{\prime \prime}}:=\left(Z_{k+1}, \ldots, Z_{n}, Z_{n+l+1}, \ldots, Z_{m}\right)$. By (3.11), this leads one to

$$
\begin{aligned}
C(\Omega) \leq & \frac{\int_{\Omega}\left|\nabla_{L} \phi\right|^{p}\left|x^{\prime}\right|^{\beta-p \alpha} d_{1}^{(\alpha+1) p-\gamma} d x d y}{\int_{\Omega}|\phi|^{p} \frac{\left|x^{\prime}\right|^{\beta}}{d_{1}^{\gamma}} d x d y} \\
\leq & (1-\lambda)^{1-p} \frac{\int_{\Omega}\left|\nabla_{L^{\prime}} v\right|^{p}|\omega|^{p}\left|x^{\prime}\right|^{\beta-p \alpha} d_{1}^{(\alpha+1) p-\gamma} d x d y}{\int_{\Omega}|v \omega|^{p} \frac{\left|x^{\prime}\right|^{\beta}}{d_{1}^{\gamma}} d x d y} \\
& +\lambda^{1-p} \frac{\int_{\Omega}|v|^{p}\left|\nabla_{L^{\prime \prime}} \omega\right|^{p}\left|x^{\prime}\right|^{\beta-p \alpha} d_{1}^{(\alpha+1) p-\gamma} d x d y}{\int_{\Omega}|v \omega|^{p} \frac{\left|x^{\prime}\right|^{\beta}}{d_{1}^{\gamma}} d x d y} \\
\leq & (1-\lambda)^{1-p} \frac{\int_{\mathbb{R}^{n-k+(m-l)}}|\omega|^{p} d z_{2} \int_{\mathbb{R}^{k+l}}\left|\nabla_{L^{\prime}} v\right|^{p}\left|x^{\prime}\right|^{\beta-p \alpha} d_{1}^{(\alpha+1) p-\gamma} d z_{1}}{\int_{\mathbb{R}^{n-k+(m-l)}}|\omega|^{p} d z_{2} \int_{\mathbb{R}^{k+l}}|v|^{p} \frac{\left|x^{\prime}\right|^{\beta}}{d_{1}^{\gamma}} d z_{1}} \\
& +\lambda^{1-p} \frac{\int_{\mathbb{R}^{n-k+(m-l)}}\left|\nabla_{L^{\prime \prime}} \omega\right|^{p} d z_{2} \int_{\mathbb{R}^{k+l}}|v|^{p}\left|x^{\prime}\right|^{\beta-p \alpha} d_{1}^{(\alpha+1) p-\gamma} d z_{1}}{\int_{\mathbb{R}^{n-k+(m-l)}}|\omega|^{p} d z_{2} \int_{\mathbb{R}^{k+l}}|v|^{p} \frac{\left|x^{\prime}\right|^{\beta}}{d_{1}^{\gamma}} d z_{1}} \\
= & (1-\lambda)^{1-p} \frac{\int_{\mathbb{R}^{k+l}}\left|\nabla_{L^{\prime}} v\right|^{p}\left|x^{\prime}\right|^{\beta-p \alpha} d_{1}^{(\alpha+1) p-\gamma} d z_{1}}{\int_{\mathbb{R}^{k+l}}|v|^{p} \frac{\left|x^{\prime}\right|^{\beta}}{d_{1}^{\gamma}} d z_{1}} \\
& +\lambda^{1-p} \frac{\int_{\mathbb{R}^{n-k+(m-l)}}\left|\nabla_{L^{\prime \prime}}\right|^{p} d z_{2}}{\int_{\mathbb{R}^{n-k+(m-l)}}|\omega|^{p} d z_{2}} \cdot \frac{\int_{\mathbb{R}^{k+l}}|v|^{p}\left|x^{\prime}\right|^{\beta-p \alpha} d_{1}^{(\alpha+1) p-\gamma} d z_{1}}{\int_{\mathbb{R}^{k+l}}|v|^{p} \frac{\left|x^{\prime}\right|^{\beta}}{d_{1}^{\gamma}} d z_{1}} .
\end{aligned}
$$

From (3.6), we get

$$
\inf _{\omega \in C_{0}^{\infty}\left(\mathbb{R}^{n-k+(m-l)}\right), \omega \neq 0} \frac{\int_{\mathbb{R}^{n-k+(m-l)}}\left|\nabla_{L^{\prime \prime}} \omega\right|^{p} d z_{2}}{\int_{\mathbb{R}^{n-k+(m-l)}}|\omega|^{p} d z_{2}}=0 .
$$

For $0<\lambda<1$, we have

$$
\begin{aligned}
C(\Omega) & \leq \inf _{\phi \in C_{0}^{\infty}(\Omega), \phi \neq 0} \frac{\int_{\Omega}\left|\nabla_{L} \phi\right|^{p}\left|x^{\prime}\right|^{\beta-p \alpha} d_{1}^{(\alpha+1) p-\gamma} d x d y}{\int_{\Omega}|\phi|^{p} \frac{\left|x^{\prime}\right|^{\beta}}{d_{1}^{\gamma}} d x d y} \\
& \leq(1-\lambda)^{1-p} \inf _{v \in C_{0}^{\infty}\left(\mathbb{R}^{k+l}\right), v \neq 0} \frac{\int_{\mathbb{R}^{k+l}}\left|\nabla_{L^{\prime}} v\right|^{p}\left|x^{\prime}\right|^{\beta-p \alpha} d_{1}^{(\alpha+1) p-\gamma} d z_{1}}{\int_{\mathbb{R}^{k+l}}|v|^{p} \frac{\left|x^{\prime}\right|^{\beta}}{d_{1}^{\gamma}} d z_{1}} .
\end{aligned}
$$

We choose $Q=k+(\alpha+1) l$ in the best constant $c_{Q, p, \beta, \gamma}^{p}$ of (1.2), and let $\lambda \rightarrow 0$ in (3.12), hence

$$
C(\Omega) \leq c_{k, l, p, \beta, \gamma}^{p} \text {. }
$$


The theorem is proved.

Proof of Corollary 1. Choosing $(\beta, \gamma)=(\alpha p,(\alpha+1) p),(\beta, \gamma)=(0, p)$ and taking $(\beta, \gamma)=(-p, 0)$ in (3.1), we get inequalities (3.2), (3.3) and (3.4) respectively. The proof of the corollary is complete.

Remark 3. We can also select $h=d_{1, \epsilon}^{1-p}\left|\nabla_{L^{\prime}} d_{1, \epsilon}\right|^{p-2} \nabla_{L^{\prime}} d_{1, \epsilon}$ to prove (3.2), as in the proof of Theorem 2.

Remark 4. In (3.1), by choosing $\beta=\alpha p, \gamma=\alpha p-\lambda, \lambda \in \mathbb{R}$, we obtain

$$
c_{k, l, p, \lambda}^{p} \int_{\Omega}|u|^{p}\left(\frac{\left|x^{\prime}\right|}{d_{1}}\right)^{\alpha p} d_{1}^{\lambda} d x d y \leq \int_{\Omega}\left|\nabla_{L} u\right|^{p} d_{1}^{p+\lambda} d x d y,
$$

where $c_{k, l, p, \lambda}=\frac{|k+(\alpha+1) l+\lambda|}{p}$. If $p=k+(\alpha+1) l$, and $\lambda<-1$, we let $\tilde{c}_{k, l, p, \lambda}=$ $\frac{|1+\lambda|}{p}, \Omega=\left\{\left(x^{\prime}, y^{\prime}, x^{\prime \prime}, y^{\prime \prime}\right) \in \mathbb{R}^{k+l} \times \mathbb{R}^{n-k+(m-l)} \mid d_{1}<R\right\}$, and then have

$$
\tilde{c}_{k, l, p, \lambda}^{p} \int_{\Omega}|u|^{p}\left(\frac{\left|x^{\prime}\right|}{d_{1}}\right)^{\alpha p}\left(\ln \left(\frac{R}{d_{1}}\right)\right)^{\lambda} d x d y \leq \int_{\Omega}\left|\nabla_{L} u\right|^{p}\left(\ln \left(\frac{R}{d_{1}}\right)\right)^{p+\lambda} d x d y .
$$

In particular

$$
\left(\frac{p-1}{p}\right)^{p} \int_{\Omega} \frac{|u|^{p}}{\left(d_{1} \ln \left(\frac{R}{d_{1}}\right)\right)^{p}}\left(\frac{\left|x^{\prime}\right|}{d_{1}}\right)^{\alpha p} d x d y \leq \int_{\Omega}\left|\nabla_{L} u\right|^{p} d x d y .
$$

This result is more general than D'Ambrosio's in [4].

Remark 5. We note that if $k=n$ and $l=m$, then (3.1) coincides with (1.2).

Theorem 3. Let $1<p<k$. Then, for every $u \in D^{1, p}(\Omega)$, there exists a constant $b_{k, p}=\frac{k-p}{p}$ such that the following inequalities hold:

$$
\begin{aligned}
& b_{k, p}^{p} \int_{\Omega} \frac{|u|^{p}}{\left|x^{\prime}\right|^{p}} d x d y \leq \int_{\Omega}\left|\nabla_{L} u\right|^{p} d x d y, \\
& b_{k, p}^{p} \int_{\Omega} \frac{|u|^{p}}{\left|d_{1}\right|^{p}} d x d y \leq \int_{\Omega}\left|\nabla_{L} u\right|^{p} d x d y .
\end{aligned}
$$

In particular, if $p=2$ and $k \geq 3$, we have

$$
\left(\frac{k-2}{2}\right)^{2} \int_{\Omega} \frac{u^{2}}{d_{1}^{2}} d x d y \leq\left(\frac{k-2}{2}\right)^{2} \int_{\Omega} \frac{u^{2}}{\left|x^{\prime}\right|^{2}} d x d y \leq \int_{\Omega}\left|\nabla_{L} u\right|^{2} d x d y .
$$

Proof. Let us first set

A simple calculation shows that

$$
h_{\epsilon}^{2}=\frac{1}{r_{\epsilon}^{p}}\left(\begin{array}{c}
x^{\prime} \\
0
\end{array}\right) .
$$

$$
\operatorname{div}_{L^{\prime}}^{\epsilon} h_{\epsilon}^{2}=\frac{1}{r_{\epsilon}^{p}}\left(k-p \frac{\left|x^{\prime}\right|^{2}}{r_{\epsilon}^{2}}\right)
$$


and

$$
\left|h_{\epsilon}^{2}\right|=\frac{\left|x^{\prime}\right|}{r_{\epsilon}^{p}} .
$$

Since $p<k$, we get $\operatorname{div}_{L^{\prime}}^{\epsilon} h_{\epsilon}^{2}>0$. From (3.5) we have

$$
\begin{aligned}
\int_{\Omega} \frac{|u|^{p}}{r_{\epsilon}^{p}} & \left(k-p \frac{\left|x^{\prime}\right|^{2}}{r_{\epsilon}^{2}}\right) d x d y=\int_{\Omega_{2}} d z_{2} \int_{\Omega_{1}} \frac{|u|^{p}}{r_{\epsilon}^{p}}\left(k-p \frac{\left|x^{\prime}\right|^{2}}{r_{\epsilon}^{2}}\right) d z_{1} \\
& \leq \int_{\Omega_{2}} d z_{2} \int_{\Omega_{1}}\left(\frac{\left|x^{\prime}\right|}{r_{\epsilon}^{p}}\right)^{p}\left[\frac{1}{r_{\epsilon}^{p}}\left(k-p \frac{\left|x^{\prime}\right|^{2}}{r_{\epsilon}^{2}}\right)\right]^{-(p-1)}\left|\nabla_{L^{\prime}} u\right|^{p} d z_{1} \\
& \leq \int_{\Omega} \frac{\left|x^{\prime}\right|^{p}}{r_{\epsilon}^{p}\left(k-p \frac{\left|x^{\prime}\right|^{2}}{r_{\epsilon}^{2}}\right)^{(p-1)}}\left|\nabla_{L} u\right|^{p} d z_{1} .
\end{aligned}
$$

Using the Lebesgue dominated convergence theorem and putting $\epsilon \rightarrow 0$ in the estimate above, we obtain (3.13). Using $\left|x^{\prime}\right| \leq d_{1}$ and (3.13), we get

$$
\int_{\Omega} \frac{|u|^{p}}{d_{1}^{p}} d x d y \leq \int_{\Omega} \frac{|u|^{p}}{\left|x^{\prime}\right|^{p}} d x d y .
$$

Hence (3.14) is obtained.

Remark 6. From the result above, it follows that the best constants in (3.13) and (3.14) lie in the interval $\left[(k-p)^{p} p^{-p},(k+(\alpha+1) l-p)^{p} p^{-p}\right]$.

As a consequence of (3.5), we give a Poincaré inequality for the vector fields on domains $\Omega$ contained in a slab. More precisely, we have

Corollary 2. Let $\Omega \subset \mathbb{R}^{n+m}$ be an open subset. Suppose that there exist $R>$ $0, s \in \mathbb{R}$ and an integer $j: 1 \leq j \leq n$, such that for any $(x, y) \in \Omega$, it follows that $\left|x_{j}-s\right| \leq R$. Then, for every $u \in C_{0}^{1}(\Omega)$, we have

$$
c \int_{\Omega}|u|^{p} d x d y \leq \int_{\Omega}\left|\nabla_{L} u\right|^{p} d x d y .
$$

where $c=(p R)^{-p}$.

The proof of the corollary follows from Theorem 3 by using the vector field defined by the formula

$$
h:=\left(\begin{array}{c}
0 \\
x_{i}-s \\
0
\end{array}\right)
$$

\section{Acknowledgement}

The authors thank Dr. Han Yazhou for many suggestions. 


\section{REFERENCES}

[1] M. Badiale and G. Tarantello, "A Sobolev-Hardy inequality with applications to a nonlinear elliptic equation arising in astrophysics," Arch. Ration. Mech. Anal., vol. 163, no. 4, pp. 259-293, 2002.

[2] W. Beckner, "On the Grushin operator and hyperbolic symmetry," Proc. Amer. Math. Soc., vol. 129, no. 4, pp. 1233-1246, 2001.

[3] L. D'Ambrosio, "Hardy inequalities related to Grushin type operators," Proc. Amer. Math. Soc., vol. 132, no. 3, pp. 725-734, 2004.

[4] L. D'Ambrosio, "Hardy-type inequalities related to degenerate elliptic differential operators," Ann. Sc. Norm. Super. Pisa Cl. Sci. (5), vol. 4, no. 3, pp. 451-486, 2005.

[5] L. D'Ambrosio and S. Lucente, "Nonlinear Liouville theorems for Grushin and Tricomi operators," J. Differential Equations, vol. 193, no. 2, pp. 511-541, 2003.

[6] N. Garofalo, "Unique continuation for a class of elliptic operators which degenerate on a manifold of arbitrary codimension," J. Differential Equations, vol. 104, no. 1, pp. 117-146, 1993.

[7] R. Monti and D. Morbidelli, "Kelvin transform for Grushin operators and critical semilinear equations," Duke Math. J., vol. 131, no. 1, pp. 167-202, 2006.

[8] P. Niu, J. Dou, and H. Zhang, "Nonexistence of weak solutions for the p-degenerate subelliptic inequalities constructed by generalized Baouendi-Grushin vector fields," Georgian Math. J., vol. 12, no. 4, pp. 727-742, 2005.

[9] S. Secchi, D. Smets, and M. Willem, "Remarks on a Hardy-Sobolev inequality," C. R. Math. Acad. Sci. Paris, vol. 336, no. 10, pp. 811-815, 2003.

[10] H. Q. Zhang and P. C. Niu, "Picone identity and Hardy inequality for a class of vector fields," $J$. Math. (Wuhan), vol. 23, no. 1, pp. 121-125, 2003.

\section{Authors' addresses}

\section{Pengcheng Niu}

Northwestern Polytechnical University, Department of Applied Mathematics, 710072, Xi' an, Shaanxi, China

E-mail address: pengchengniulyahoo.com.cn

\section{Jingbo Dou}

Northwestern Polytechnical University, Department of Applied Mathematics, 710072, Xi'an, Shaanxi, China

E-mail address: djb76eeyou.com 